\title{
High spatial resolution imaging of the star with a transiting planet WASP-33 (Research Note)
}

\author{
A. Moya ${ }^{1}$, H. Bouy ${ }^{1}$, F. Marchis ${ }^{2}$, B. Vicente ${ }^{3}$, and D. Barrado ${ }^{4,1}$ \\ 1 Departamento de Astrofísica, Centro de Astrobiología (INTA-CSIC), PO Box 78, 28691 Villanueva de la Cañada, Madrid, Spain \\ e-mail: amoya@cab.inta-csic.es \\ 2 Department of Astronomy at UC-Berkeley, 601 Campbell Hall, Berkeley CA 94720 USA \\ and Carl Sagan Center at the SETI Institute, 189 Bernado Av, Mountain View CA 94043, USA \\ 3 Instituto de Astrofísica de Andalucía (CSIC), Glorieta de la Astronomía S/N, 18008 Granada, Spain \\ ${ }^{4}$ German-Spanish Astronomical Center, Observatorio de Calar Alto, C/ Jesús Durban Remon 2-2, 04004 Almeria, Spain
}

Received 14 March 2011 / Accepted 12 October 2011

\begin{abstract}
Context. The planetary system WASP-33 is an interesting target because of the discovery of a transiting planet, the misalignment of the stellar rotation axis and the planet orbit, the possible existence of an additional planet in the system, and the presence of $\delta$ Scuti pulsations in the hosting star. Its study promises in particular to help us understand the origin and evolution of giant planets orbiting very close to a star.

Aims. We analyse high spatial resolution imaging with an adaptive optics system on the W.M. $10 \mathrm{~m}$-Keck II telescope to investigate the prediction of an additional planet, and any other objects, and constrain the possible formation scenarios of the system.

Methods. In November 2010, we recorded high spatial resolution images from 1 to $2.5 \mu \mathrm{m}$ using the W.M. $10 \mathrm{~m}$-Keck II telescope and its adaptive optics system, obtaining broad $(J c, H c$, and $K c)$ and narrow-band (FeII) images of the system. After data reduction, the contrast and angular resolution provided by this instrument allowed us to constrain the multiplicity of this system and detect one potential companion.

Results. We present the discovery of a new object at a distance of $1.961^{\prime \prime} \pm 0.003^{\prime \prime}$ from the WASP-33, at a position angle of 276.32 $\pm 0.24 \mathrm{deg}$. It might be either a dwarf star or brown dwarf, or an extragalactic object. In the case of a galactic object and assuming the same distance from the Sun, it would be 227 AU from the central star. We find no evidence of any additional objects. This constrains the possible constituents of the system, depending on its age and the constituent masses.

Conclusions. We find a potential companion object to WASP-33. We need to confirm the gravitational link, but this object could influence the evolution of the planetary system depending on its orbital eccentricity. We exclude the existence of any additional objects. For example, objects of $0.8 M_{\odot}$ at projected physical distances greater than [2, 5] AU, objects of $0.3 M_{\odot}$ at projected physical distances greater than $[11,18] \mathrm{AU}$, or objects of $0.072 M_{\odot}$ at projected physical distances greater than $[18,75] \mathrm{AU}$, depending on the age of the system.
\end{abstract}

Key words. instrumentation: adaptive optics - techniques: image processing - techniques: high angular resolution - binaries: visual - stars: individual: WASP-33 - stars: individual: HD 15082

\section{Introduction}

The discovery of hundreds of exoplanets in the past few years, as a result of the increasing number of observational techniques and their accuracies, has opened a number of questions about the origin and evolution of planetary systems. The number of theories developed to explain the observational findings has also increased in a similar way. Owing to observational biases, a large number of massive gas planets orbiting close to the hosting star have been reported. This has stimulated the development of several theories explaining the assumed planet migration, including disk-planet interaction models (Ida \& Lin 2004), planet-planet scattering models (Nagasawa et al. 2008; Chatterjee et al. 2008), or Kozai mechanism plus tidal dissipation models (Wu et al. 2007). The alignment of the planet's orbit and the stellar rotation axis, measured mainly using the Rossiter-McLaughlin effect, is a key to disentangling, and discriminating between the different formation and migration scenarios. Thanks to the most recent observational data, the misalignment found in some systems can be explained by the presence of other massive bodies (Narita et al. 2010), or can be related to the stellar effective temperature, hence its internal structure (Winn et al. 2010). This last prediction suggests a link between the planet-star misalignment and the absence of an outer highly developed convective zone, that is, it is restricted to stars with $T_{\text {eff }}>6200^{\mathrm{O}} \mathrm{K}$, approximately. The study of hot stars with planets is imperative to the understanding of these scenarios.

The discovery of exoplanets around A stars using radial velocity (RV) data is not an easy task. The mass of these stars only allows the detection of massive planets orbiting close to the star. The star WASP-33 (HD15082, $V=8.33, v \sin i=$ $86 \mathrm{~km} \mathrm{~s}^{-1}$, Christian et al. 2006) is the only $A$ star known to have a transiting planet. This planet has been confirmed using RV (Collier-Cameron et al. 2010), which means that the planet is massive. In this latter work, the authors: 1) refine the stellar and planetary physical parameters; 2) confirm the orbital period 
of the planet (1.22 d) and; 3 ) report a misalignment between the stellar and orbital spin axis. In addition, they suggest possible stellar $\delta$ Scuti pulsations, something that is unsurprising because the star is located in the HR $\delta$ Scuti instability strip (Grigahcène et al. 2010). These pulsations were confirmed by Herrero et al. (2011), and can help in understanding the internal structure of the star. Herrero et al. (2011) found a coincidence between the pulsating period and a multiple of the orbital period. All these characteristics make WASP-33 a very interesting object from the point of view of both giant planet formation and evolution and stellar structure and evolution.

The presence of additional giant planets orbiting the hosting star would help us to understand the planet formation scheme. In Collier-Cameron et al. (2010), the possible existence of an additional object was suggested by taking into account the RV residuals after the planet and pulsation analysis. In the present study, we searched for additional WASP-33 companion objects using direct imaging.

\section{Observations and data processing}

We observed WASP-33 using the adaptive optics system of the Keck-II telescope and its NIRC2 infrared camera $(1024 \times$ 1024 pixels). With a $V$-magnitude of 8.3 , the star is bright enough to be used as a reference of the wavefront analysis. The data collected on November 292010 were recorded in $J c$ (bandwidth of $0.020 \mu \mathrm{m}$ centred on $1.213 \mu \mathrm{m}$ ), $H c$ (bandwidth of $0.023 \mu \mathrm{m}$ centred on $1.580 \mu \mathrm{m}$ ), and $K c$ (bandwidth of $0.030 \mu \mathrm{m}$ centred on $2.271 \mu \mathrm{m}$ ), under good seeing conditions but high humidity. For each filter, we recorded three images at different positions of the detector. Each image is composed of five in $J c$, five in $H c$, and twenty in $K c$ co-added frames with an individual exposure time of $2.9 \mathrm{~s}$. The angular resolution in $K c$-band is estimated to be 55 milli-arcsec (mas), which is very close to the diffraction limit of the telescope (47 mas). Additional observations were recorded on November 30 in $K c$ and FeII (bandwidth of $0.026 \mu \mathrm{m}$ centred on $1.645 \mu \mathrm{m}$ ) filters. The data set is identical with three images composed of six in $K c$ and twenty in FeII co-added frames with an individual exposure time of $3 \mathrm{~s}$. Since the atmospheric conditions were not optimal (fog and high altitude cirrus) the angular resolution in $K c$ band is estimated in 100 mas. For both nights, the observations were performed at $\sim 10$ UT with an optimal low air-mass of 1.1. We estimated the seeing at the beginning of the first night to be $\sim 0.7^{\prime \prime}$ in visible. The Strehl ratio was estimated to be $\sim 40 \%$ on the first night using a $K c$ simulated PSF.

The data were processed using standard procedures with the Eclipse software package (Devillard 1997). The individual images were flat-field corrected and then registered and median combined. Aperture photometry of the two sources present in the field was extracted using the $\operatorname{IRAF}^{1}$ daophot package. An aperture of 20 pixels and a sky annulus between $20-25$ pixels were used. The flux ratio of the bright primary to the wide companion was computed, and the absolute magnitude of the wide companion was derived in each of the broad-band filters using the 2MASS magnitudes of the primary (Skrutskie et al. 2006).

No astrometric standard field was obtained during the night, and the NIRC2 pixel scale and orientation cannot be accurately calibrated. Ghez et al. (2008) found an average platescale of

\footnotetext{
1 IRAF is distributed by the National Optical Astronomy Observatory, which is operated by the Association of Universities for Research in Astronomy (AURA) under cooperative agreement with the National Science Foundation.
}

Table 1. Photometric colours observed for WASP-33 and the difference between the object discovered and WASP-33 in the filters $J c, H c$, and $K c$.

\begin{tabular}{|c|c|c|c|c|}
\hline Object & $\overline{\text { Band }}$ & 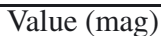 & Error (mag) & Source \\
\hline \multirow{3}{*}{ WASP-33 } & $J$ & 7.581 & 0.021 & \multirow{3}{*}{ 2MASS } \\
\hline & $H$ & 7.516 & 0.024 & \\
\hline & $K \mathrm{~s}$ & 7.468 & 0.024 & \\
\hline \multirow{6}{*}{$\begin{array}{l}\text { New } \\
\text { (candidate) }\end{array}$} & $\Delta J c$ & 6.62 & 0.03 & \multirow{6}{*}{ This work } \\
\hline & $\Delta H c$ & 6.13 & 0.02 & \\
\hline & $\Delta K c$ & 5.69 & 0.01 & \\
\hline & $\Delta J c-\Delta K c$ & 1.04 & 0.04 & \\
\hline & $\Delta J c-\Delta H c$ & 0.55 & 0.05 & \\
\hline & $\Delta H c-\Delta K c$ & 0.49 & 0.03 & \\
\hline
\end{tabular}

$9.963 \pm 0.006 \mathrm{mas} /$ pixel over the period 2004 and 2008. We adopted this value for our measurements. The uncertainty in the position angle of the camera on the sky is also unknown. It is determined mostly by the required engineering adjustments, which can introduce slight rotations in the camera. Ghez et al. (2008) found systematic offsets of up to $0.2 \mathrm{deg}$ with respect to the rotation keywords given by the control system. This offset was also added to the measurement uncertainties. No distortion correction was applied to our measurements.

\section{Result 1: A new companion candidate to WASP-33}

In Fig. 1, the final image after basic data-processing is shown in the $K c$-band. Here we can see the presence of an object $1.961^{\prime \prime}$ $\pm 0.003^{\prime \prime}$ distant from the star and at a position angle of 276.32 $\pm 0.24 \mathrm{deg}$. The uncertainty was computed from the $1 \sigma$ dispersion in the individual measurements in each filter plus the above-mentioned instrumental uncertainties (platescale and position angle of the camera on the sky). No other object was found by visual inspection. This is an unknown object. The first part of this study is devoted to the analysis of this object and its possible membership to the WASP-33 system. Table 1 shows the photometric colours observed, including those of WASP-33 as a reference. No photometric standard star was obtained during the night. To assess the nature of the companion, we hereafter assume that the flux ratio in the $J c, H c$, and $K c$ Mauna Kea filters can be applied to the corresponding 2MASS filter system. Therefore, in the absence of confusing issues, we use the 2MASS filters notation to label our results when WASP-33 and the new candidate are compared.

With these limitations in mind, we find that the $H-K$ s value observed for this object suggests a spectral type compatible with either a dwarf star/brown dwarf, a giant star, or an extragalactic object. With the photometric data available, the precise determination of the spectral type, or any additional classification, is not an easy task. Nevertheless, we are able to ascertain whether the object is a giant star. In Fig. 2, we see that the observed object data is compatible with either a dwarf star or brown dwarf, and not a giant star in the $H-K$ s versus $J-H$ diagram, although an extragalactic object cannot be ruled-out.

To check whether the two sources might be coeval, we compare their luminosities and colours with different theoretical isochrones (Siess et al. 2000). Figure 3 shows a $J-K$ s vs. $J$ diagram, assuming that the two sources are at the same distance $(116 \pm 11 \mathrm{pc}$; van Leeuwen 2007). The figure shows that WASP 33 is an early-type star with an age of 10-500 Myr, which is compatible with the spectroscopic estimation led by Cameron et al. (2010). It also shows that the NIR photometry 


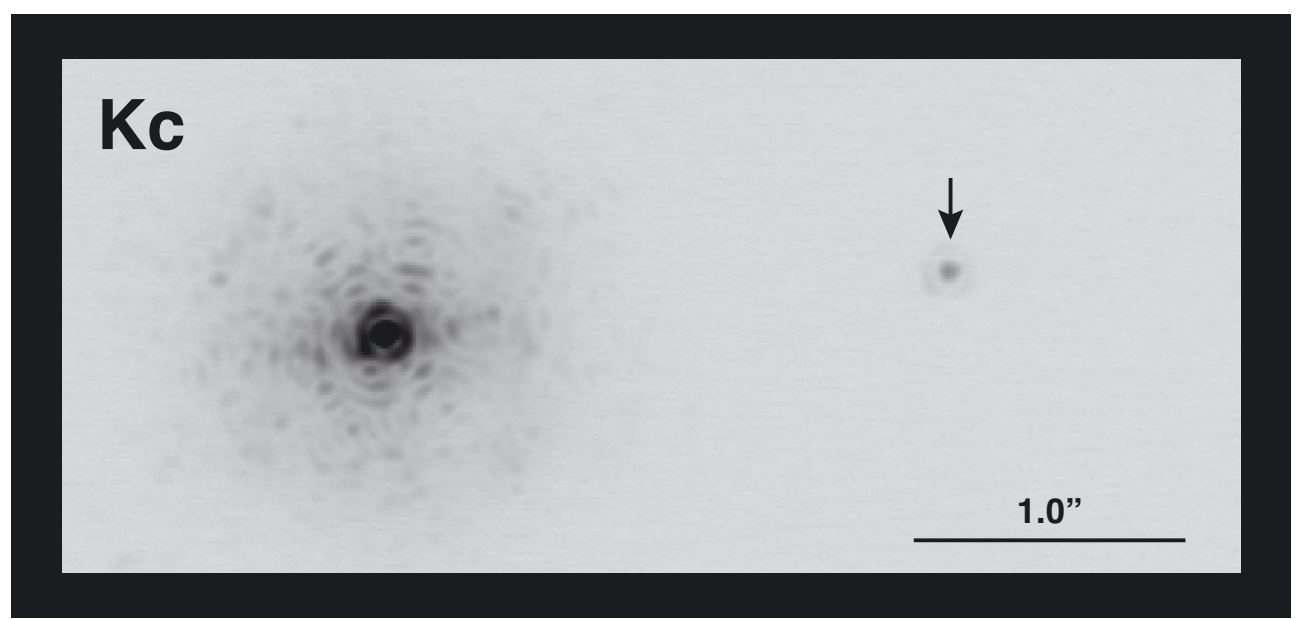

Fig. 1. Direct imaging of WASP-33 in the $K c$-band. The new object, located at $\sim 2$ arcsec is marked with an arrow.

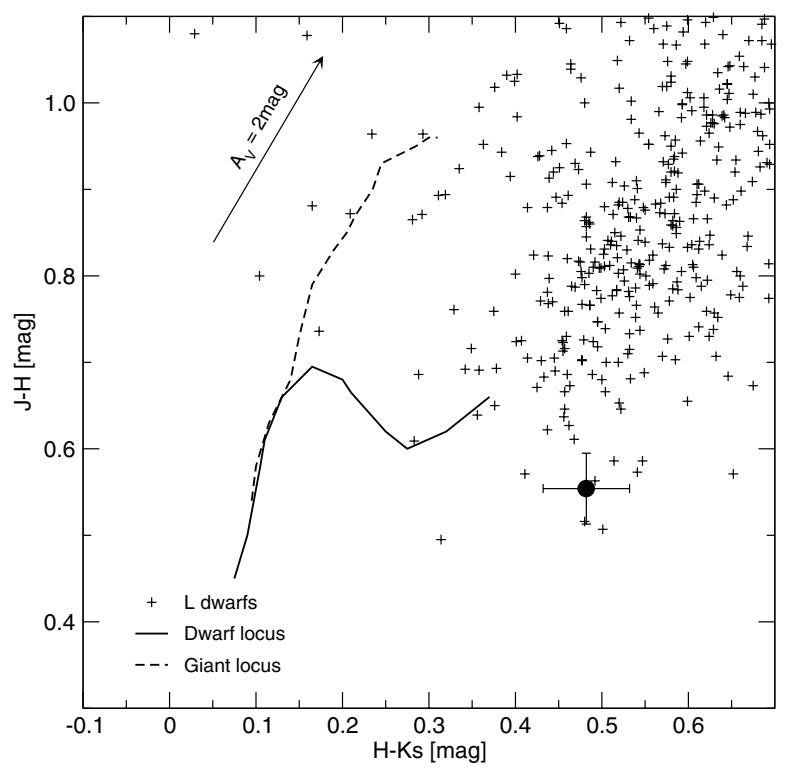

Fig. 2. $H-K$ s vs. $J-H$ comparison of the observed companion object with those predicted for giant and dwarf stars. A set of known L-type dwarfs is also shown (crosses).

of the candidate is that expected for a bound low-mass star, or a substellar companion, of the same age as WASP-33, although the age of the companion is poorly constrained by photometry, an age of 1500 Myr also being possible. Contamination by a foreground or background object cannot be excluded based on our photometry, except in the case of background giants. We note that the isochrone of $100 \mathrm{Myr}$ is close to the centre of the error boxes of both objects, making this the most probable age for both (assuming the same distance).

Confirmation of the association of WASP-33 and its tentative companion could be made astrometrically by showing that both are common proper motion objects. The orbital motion of the new object would be negligible, since, at a distance of $116 \mathrm{pc}$, the projected separation of the companion is $227 \mathrm{AU}$, implying an orbital period of thousands of years.

From the revised Hipparcos catalogue (van Leeuwen 2007), the absolute proper motion of WASP-33 is $\left(\mu_{\alpha} \cos \delta=\right.$

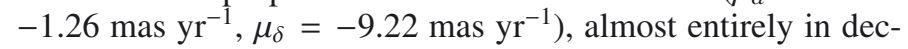
lination, and the object is almost due west (positional angle $276 \mathrm{deg}$ ). Thus, if the object were a distant background star with negligible absolute proper motion, then similar observations

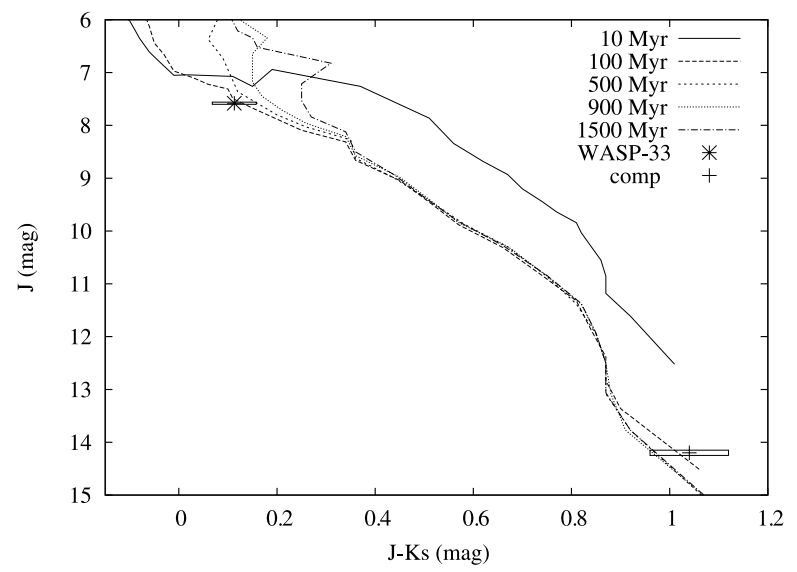

Fig. 3. $J-K$ s vs. $J$ comparison of WASP-33 and the observed companion object with different isochrones.

made in one year's time would show that WASP-33 had moved in declination (south) by 9.2 mas, while the object remain still. Geometrically, the expected change in one year with the relative position of WASP-33, and an object with a hypothesised motion of zero, would be almost entirely in position angle, $0.27 \mathrm{deg}$, while $0.001^{\prime \prime}$ in separation.

Therefore, with an uncertainty in position angle of $0.24 \mathrm{de}-$ grees, $\mathrm{a} \sim 3 \sigma$ detection of the relative proper motion of the possible companion and WASP-33 could be made using data with a three-year baseline, if the tentative companion were instead a low proper motion background star. If the tentative companion were a faint foreground star, with a consequently large proper motion, a non-zero relative proper motion would be even more obvious. Conversely, a zero relative position in three years would indicate a common proper motion and confirm that the two objects are physical associated with one another.

Finally, if the newly discovered object is a companion of WASP-33, its mass is in the range $[<0.1,0.2] M_{\odot}$ and its $T_{\text {eff }}=$ $3050 \pm 250 \stackrel{0}{ }-K$. These ranges were estimated by comparing the observed $J-K$ s vs. $J$ photometry with the isochrones used for Fig. 3 (Siess et al. 2000), with ages in the range [10, 1500] Myr.

\section{Result 2: No additional objects in the image}

The absence of additional objects in the image obtained offers clear constraints on the presence of very low mass stars or brown dwarfs orbiting WASP-33. In Fig. 4, we show the sensitivity of 


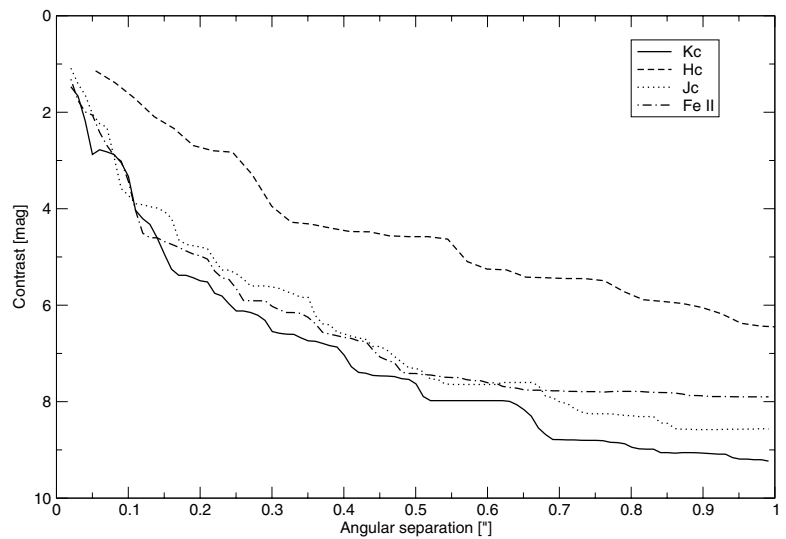

Fig. 4. Sensitivity of the present observations for different filters as a function of the angular separation. The solid line is the observational limit obtained with the $\mathrm{Kc}$-filter, respectively, with the dash line - $\mathrm{Hc}$ filter, dot line $-J c$-filter, and dash-dot line - Fe II filter.

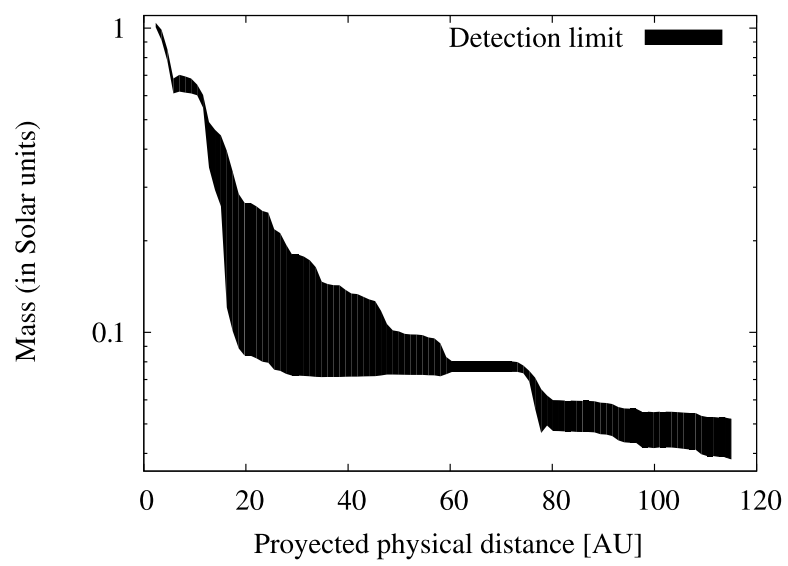

Fig. 5. Maximum mass in solar units of possible objects beyond the detection limits of our observations as a function of the projected physical distance to the central star (in AU). The observational errors in the determination of the magnitude, distance, and age have been taken into account. See text for details.

the present observations for different filters, as a function of the angular separation. The solid line is the observational limit obtained with, the $K c$-filter, the dash line the $H c$-filter, the dotted line the $J c$-filter, and the dash-dot line the Fe II filter. The $K c$-filter offers the highest accuracy and the presence of different objects can be ruled-out as a function of the distance to WASP-33. Figure 5 shows an estimate of this limit (using the $K c$-filter as a reference) in terms of the mass in solar units and distance from the star in AU for the age determined for WASP33. The isochrones used to convert luminosities into masses are made from the latest NextGen models (Baraffe et al. 1997) down to $2.300^{\mathrm{O}} \mathrm{K}$, and DUSTY models (Baraffe et al. 2002) up to $2.300 \mathrm{O} \mathrm{K}$. The limiting region is bounded by the errors in the determination of the magnitude of WASP-33, its distance, and its age. The age of the objects is assumed to be in the range [10, 500] Myr, that is, from the ZAMS to the oldest age estimated for WASP-33. The discontinuities in the lower boundary of the limiting band are a reflection of the small discontinuity found in the transition between the two models used.

In this figure, for example, we determine that the existence of objects of $0.8 M_{\odot}$ can be ruled-out at projected physical distances larger than $[2,5]$ AU. The same can be said for $0.3 M_{\odot}$ stars and projected physical distances larger than [11, 18] AU. Substellar objects (of masses lower than $0.072 M_{\odot}$ ) can be ruled out at projected physical distances larger than 75 AU. The lower mass limit for these objects cannot be properly estimated owing to the discontinuity described in the previous paragraph.

\section{Conclusions}

We have presented high spatial resolution images of the system WASP-33. We have obtained images from 1 to $2.5 \mu \mathrm{m}$ using the W.M. 10 m-Keck II telescope and its adaptive optics system, obtaining broad $(J c, H c$, and $K c$ ) and narrow-band (FeII) images of the system.

We have discovered a new object, located at a distance of $1.961^{\prime \prime} \pm 0.003^{\prime \prime}$ from the central star, at a position angle 276.32 $\pm 0.24 \mathrm{deg}$. The colour information obtained constrains this object to be either a dwarf star/brown dwarf, or an extragalactic object. Assuming that both objects are at identical distances from the Sun, we have calculated the distance between these objects to be $227 \mathrm{AU}$. With the observations obtained, we have also restricted the age of WASP-33 to be younger than $500 \mathrm{Myr}$, with a most probable age of $100 \mathrm{Myr}$, confirming the estimates of Collier-Cameron et al. (2010). The companion object, assuming identical distances, might have a wider range of age, and a similar most probable age. We will be able to establish whether these objects are comoving using astrometry, by performing an additional observation, with similar accuracy, in three years time.

The lack of any additional object in the image introduces constraints on the existence of other stellar companions. Thanks the sensitivity of our observations, we can exclude the existence of a star with either a mass $0.8 M_{\odot}$ at projected physical distances larger than $[2,5] \mathrm{AU}$, a mass $0.3 M_{\odot}$ at projected physical distances larger than $[11,18] \mathrm{AU}$, or a mass $0.072 M_{\odot}$ at projected physical distances larger than $[18,75] \mathrm{AU}$, for ages in the range [10, 500] Myr.

Our future goals will be the confirmation of the link between the newly discovered object and WASP-33, and the determination of its orbital parameters. With these data, we will be able to study the possible influence of the discovered companion on the orbit of the planet WASP-33b.

Acknowledgements. The authors wish to thank the referee for useful and interesting comments. A.M. warmly thanks J.A. Caballero for fruitful discussions and suggestions. A.M. acknowledges the funding of AstroMadrid (CAM S2009/ESP-1496). This research has been funded by the Spanish grants ESP2007-65475-C02-02, AYA 2010-21161-C02-02. F.M. work was supported by the National Science Foundation under award number AAG-0807468.

\section{References}

Baraffe, I., Chabrier, G., Allard, F., \& Hauschildt, P. H. 1997, A\&A, 327, 1054 Baraffe, I., Chabrier, G., Allard, F., \& Hauschildt, P. H. 2002, A\&A, 382, 563 Christian, D. J., Pollacco, D. L., Skillen, I. et al. 2006, MNRAS, 372, 1117 Cameron, A. C., Guenther, E., Smalley, B., et al. 2010, MNRAS, 407, 507 Chatterjee, S., Ford, E. B., Matsumura, S., \& Rasio, F. A. 2008, ApJ, 686, 580 Devillard, N. 1997, The Messenger, 87, 19

Ghez, A. M., Salim, S., Weinberg, N. N., et al. 2008, ApJ, 689, 1044 Grigahcène, A., Antoci, V., Balona, L., et al. 2010, ApJ, 713, L192 Herrero, E., Morales, J. C., Naves, R., \& Ribas, I. 2011, A\&A, 526, L10 Ida, S., \& Lin, D. N. C. 2004, ApJ, 616, 567

van Leeuwen, F. 2007, A\&A, 474, 653

Nagasawa, M., Ida, S., \& Bessho, T. 2008, ApJ, 678, 498

Narita, N., Kudo, T., Bergfors, C., et al. 2010, PASJ, 62, 779

Pravdo, S. H., Shaklan, S. B., Wiktorowicz, S. J., et al. 2006, ApJ, 649, 389

Siess, L., Dufour, E., \& Forestini, M. 2000, A\&A, 358, 593

Skrutskie, M. F., Cutri, R. M., Stiening, R., et al. 2006, AJ, 131, 1163

Winn, J. N., Fabrycky, D., Albrecht, S., \& Johnson, J. A. 2010, ApJ, 718, L145

Wu, Y., Murray, N. W., \& Ramsahai, J. M. 2007, ApJ, 670, 820 